\title{
A Depth Map Acquired By Integrated Imaging Based On Adaptive Matching Window
}

\author{
Xia Wen, Wang Yu* and Piao Yan \\ Electronic and information engineering institute, Changchun University of Science and Technology, \\ Changchun, China \\ muxie2002@126.com
}

Keywords: Integrated imaging, Stereo matching, View, Adaptive window, Depth map

\begin{abstract}
In this paper, we prose a method to obtain the depth map based on adaptive matching window by Integrated imaging technology. This method combines the boundary information in I1I2I3 color space and minimum threshold value of the window effectively. So that the self-adapted matching window could adjust its size and shape to improve the matching accuracy. Furthermore, in order to take advantage of multiple views of the integrated imaging, the consistency checking method is introduced into integrated imaging to reduce the false matching rate. Experimental results show that the proposed method can obtain the depth map with high accuracy.
\end{abstract}

\section{Introduction}

Depth information are widely used in the three-dimensional scene visualization, medical image processing, remote sensing and navigation. Integrated imaging is one of the three-dimensional (3D) imaging technologies based on lens array to capture incoherent light rays from different directions ${ }^{[1]}$. Stereo matching is classic problem in computer vision that obtain the depth information from view images.

The size of the matching window is an important factor affecting the quality of stereo matching ${ }^{[2]}$. Reference 10 provides a block matching based on fixed window algorithm. This method produces hypertrophy phenomenon if the size of the window is too big. Therefore, multiple window algorithm and self-adapted matching window have been proposed by many scholars. Reference 11 provides a adaptive matching window based on grey information of images without consider the color information of images, which is easy to miss the subtle boundary information.

\section{The Principle Of Stereo Matching Based On Integrated Imaging}

\section{Acquisition Of Views}
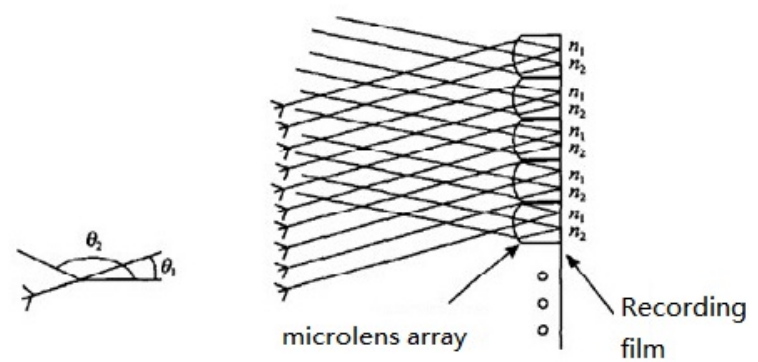

Figure. 1 the relationship between the perspective and gathering position

According to the principle of lens imaging, the parallel lights all along the same specific direction will be focused on the same point. As shown in figure 1.The formation of view is to extract pixels from the same location under each lens of the lens array. Extracting a pixel point from the sub image of the corresponding lens array will get the view with low resolution, which is not conducive to the late stereo matching between the views. In order to improve the resolution of the view, the paper extract $\mathrm{N}$ pixels from surrounding pixels of the extracted pixels that is extract $\mathrm{N} * \mathrm{~N}$ pixels in horizontal and vertical direction where $\mathrm{N}$ is within the number of observed pixels[5]. 


\section{The Calculation Of Parallax}

The similarity of matching point $(\mathrm{i}, \mathrm{j})$ in the reference image as shown in formula (1).

$$
\begin{aligned}
& d(i, j)=\underset{d \in D}{\arg \max }\{S A D(i, j, d)\} . \\
& S A D(i, j, d)=\sum_{i \in w} \sum_{j \in w} \sum_{k=1}^{3} \frac{I_{1}(i, j, k)}{\sum_{k=1}^{3} I_{1}(i, j, k)}\left|I_{1}(i, j, k)-I_{2}(i, j+d, k)\right| ; d \in[0, D] .
\end{aligned}
$$

$\mathrm{I}_{1}$ is assumed as reference images, Where $\mathrm{w}$ is matching window, $\mathrm{D}$ is the search scope of parallax, and $\mathrm{K}$ represents the $\mathrm{R}, \mathrm{G}, \mathrm{B}$ color space.

The Relationship Between The Parallax And Depth

The relationship between the parallax $d$ and depth $\mathrm{D}$ of any point in space is given by ${ }^{[6]}$ :

$D=d \bullet \varphi \bullet F / \Delta$.

Where $\mathrm{d}$ is parallax of matching point, $\varphi 、 \mathrm{~F}$ is the focal length and aperture of lens respectively and $\Delta$ is the sampling distance between two matching views.

\section{The Selection Of Adaptive Window}

\section{The Extraction Of The Boundary Information}

Building the window based on the gray information of reference image is one of adaptive window matching method. The pixel in matching image which changes dramatically are seemed as the boundary point. Then others are seemed as the non-boundary point. In order to obtain perfect outline information, we consider the I1I2I3 color information to extract the boundary information.

Because of the highly correlation between R, G, B colors, some portions with the same color will be divided into different areas[8]. I1I2I3 color space is closer to human visual and orthogonal mutually. The transforming method is given by:

$$
\left\{\begin{array}{c}
I 1=\frac{1}{3}(R+G+B) \\
I 2=\frac{1}{2}(R-B) \\
I 3=\frac{1}{4}(2 G-R-B)
\end{array}\right.
$$

We use Canny operator to get the boundary information of I1, I2, I3 color space respectively because of the high SNR value and high precision, then combines the extracted results.

\section{The Growing Mode Of The Window}

To take the matching point $(i, j)$ as center of window, search the points to the left, right, up and down in the horizontal and vertical direction until the searched point is the boundary point. The vertical ordinate at the end of the search to the left and right are denoted as $h_{l} 、 h_{r}$ respectively. The horizontal ordinate at the end of the search to the up and down are denoted as $v_{u} 、 v_{d}$ respectively. All the searching pixels are connected to form the shape of a cross.

If the matching point is belongs to non-boundary point and $v_{u} 、 v_{d}$ is far less than $h_{l}, h_{r}$, then increase the minimum supported window of the pixel that is increase the minimum value of $h_{l}$ 、 $h_{r} 、 v_{u} 、 v_{d}$. And the window re-grows according to the step (1). Otherwise, the minimum value of window is one which is obtained by step (1).

To take the other point $(\mathrm{u}, \mathrm{v})$ which is on the vertical line of cross as center of window and growing threshold is same as step (2), search the boundary point as step (1). The window of matching point $(i, j)$ is formed when search all the pixels on the vertical line. As is shown in figure 2 . 


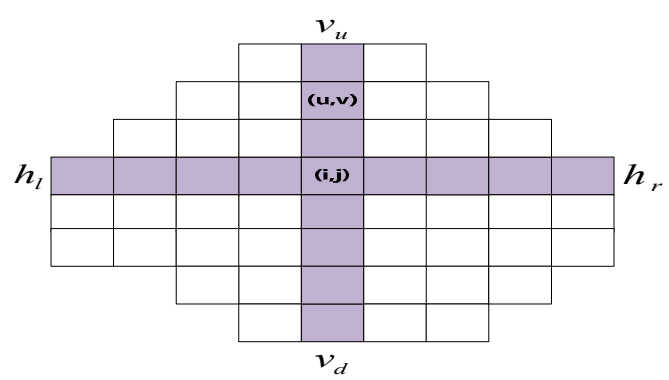

Fig.2 The adaptive window area of point $(i, j)$

\section{Parallax Correction}

The traditional consistency checking method is to use two disparity map which is obtained by two views for correction. In the paper, the traditional consistency checking method is introduced to the Integrated imaging. In order to fully take advantage of multiple views, we use three views which are extracted at a constant interval for stereo matching. Taking the middle view as a reference image and the other two views as target images to obtain two disparity maps which is named the left disparity map and the right disparity map. The parallax value of the corresponding pixels in the two views should be the same because of the the same reference image and the sampling interval. If the parallax value of the corresponding pixels in the two views are equal to each other, then denoted as correct points. Otherwise, the pixels are denoted as mismatching points.

The correction of the parallax value is conducted in the reference image. To build a adaptive supported window at the center of the mismatching point, then search the correct point with the closest color and distance. The parallax value of the mismatching point is equal to the parallax value of the searching point.

\section{The Experimental Results}
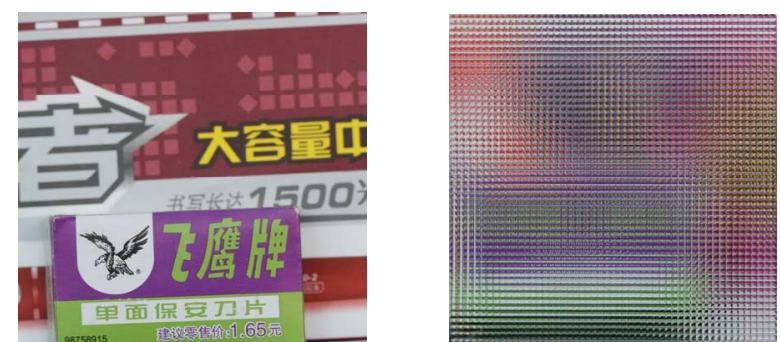

Fig.3 3D scene and elementary images array

The focal length of lens array $\mathrm{f}$ is $3 \mathrm{~mm}$, pitch of lens array $\mathrm{p}$ is $1.08 \mathrm{~mm}$. The collected elementary image include $51 * 51$ images. The resolution of each image has $40 * 40$ pixels.
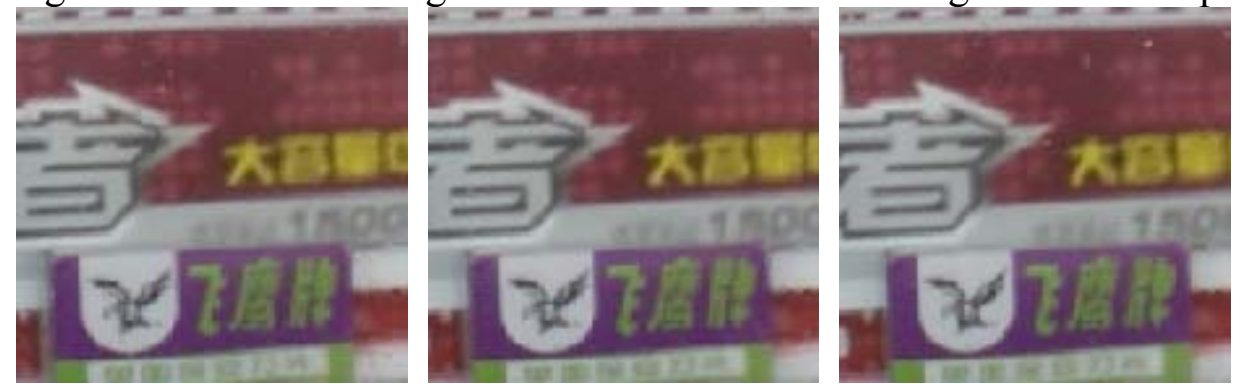

Fig.4 Extracted three views. The Extracted position are $(25,58),(25,62)$ and $(25,66)$ respectively. 


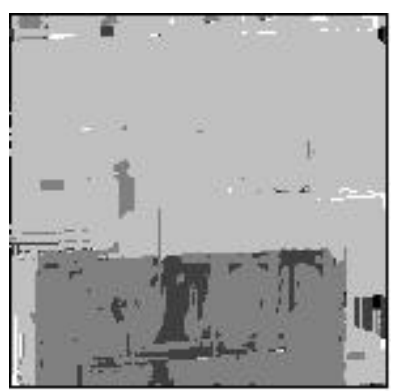

(a) left depth map

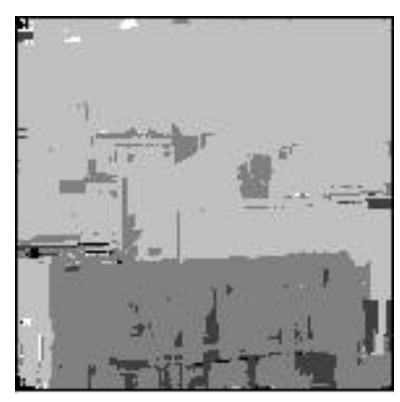

(a) right depth map

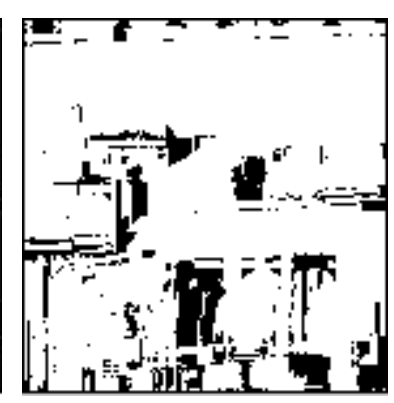

(c)mismatching points

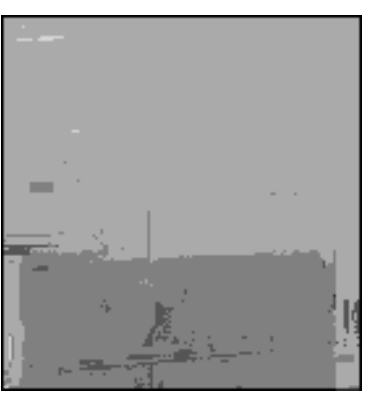

(d)corrected depth

Fig. 5 The depth map obtained by the adaptive method

\section{Summary}

The size of a matching window is an important factor affecting the quality of the stereo matching. To get a robust matching window, we extract the boundary information in I1I2I3 color space which is similar to the human visual system. In order to reduce the mismatching rate, we make use of two depth map for parallax correction which are obtained by three views. The experiment results show that the proposed method can obtain an accurate depth map.

\section{Acknowledgments}

This work was supported by a grant from the National Natural Science Foundation of China (No. 60977011), the Natural Science Foundation of Jilin Province (No. 201215142) and the Education Department of Jilin Province "twelfth Five-Year plan" Science and Technology Research Project (No. 2012-33).

\section{References}

[1] Adrian Stern, Bahram Javidi, in:Shannon number and information capacity of three-dimensional integral imaging, edited by Optical Society of America, Vol.21, No.9, p.1602-1611(2004) .

[2] Zhou Xiuzhi, Wen Gongjian and Wang Runsheng, in: Fast stereo matching based on adaptive window, edited by Journal Of Computers, Vol.29, No.3, p.473-479(2006).

[3] Men Chaoguang, Bian Jilong, in: Fast stereo matching method based on limited search space, edited by Journal Of Jinlin University(Engineering Edition), Vol.39, No.6, p.519-521(2012) .

[4] Dong-Hak Shin, Hoon Yoo, in: Image quality enhancement in 3D computational integral imaging by use of interpolation methods, edited by Optical Society of America, Vol.15, No.19, p.12039-12049(2007).

[5] Dong-Hak Shin, in: Improved Viewing Quality of 3-D Images in Computational Integral Imaging Reconstruction Based on Lenslet Array Model, edited by ETRI Journal, Vol.28, No.4, p. 521-524(2006).

[6] Wu Chunhong, You Fucheng, in: Depth measurement method based on three-dimensional panoramic image technolog, edited by Chinese Journal Of Image And Graphics, Vol.11, No.4, p. 563-569(2006).

[7] Zeng Fanzhi, Bao susu: An adaptive multi-window stereo matching algorithm, edited by Computer Science, Vol.39, No.6, p.519-521(2012).

[8] Yang Zhenya: Study of several basic problems of color image processing, edited by East China Normal University(2008). 